\title{
Active smoking is associated with severity of coronavirus disease 2019 (COVID-19): An update of a meta-analysis
}

Fei R. Guo ${ }^{1,2}$

\section{Dear Editor,}

The letter to the Editor of Lippi and Henry ${ }^{1}$ published in the European Journal of Internal Medicine and entitled 'Active smoking is not associated with severity of coronavirus disease 2019 (COVID-19)' had errors and led to the wrong conclusion.

Lippi and Henry ${ }^{1}$ searched PubMed and Web of Science up to 9 March 2020, and identified 5 studies ${ }^{2-6}$ with data on smoking and severity of COVID-19. They performed a meta-analysis revealing a pooled OR of 1.69 (95\% CI: 0.41-6.92) and concluded that active smoking does not seem to be significantly associated with enhanced risk of progressing towards severe disease in COVID-19. There were several mistakes in their data collection that led to errors in the meta-analysis. In table 1 of their letter, they indicated the outcome of Guan et al. study ${ }^{2}$ to be 'Admission to ICU, mechanical ventilation, death', however, they used the data of 'severe disease' in the study. According to the Guan et al. ${ }^{2}$ paper, the number of patients having composite outcome should be 66 , and for patients not having a composite outcome should be 1019. However, Lippi and Henry ${ }^{1}$ used 172 and 913, respectively, in their paper. This is the most serious mistake because the Guan et al. ${ }^{1}$ study contributes to most of the cases in the meta-analysis. Moreover, the non-severe patients in the Huang et al. ${ }^{3}$ study should be 28 and not 31 . The non-severe patients in the Yang et al. ${ }^{5}$ study should be 20 and not 18 . The severe patients in the Zhang et al. ${ }^{6}$ should be 58 and not 60 . The errors led to the wrong sample size of these 3 studies as well. Lippi and Henry ${ }^{1}$ were only correct in one ${ }^{4}$ out of the 5 studies.

I performed an updated meta-analysis according to the correct data using RevMan Ver. 5.3, and provide the forest plot (Figure 1). The pooled OR was 2.20 (95\% CI: $1.31-3.67 ; \mathrm{p}=0.003)$. The heterogeneity was moderate $\left(\mathrm{I}^{2}=57 \%\right)$. There was no obvious publication bias by the funnel plot. Though there are new studies published after the Lippi and Henry ${ }^{1}$ paper, the purpose of this letter is to correct their errors, therefore new studies are not included in the updated meta-analysis.

In a systemic review published by Vardavas and Nikitara ${ }^{7}, 5$ studies were included. Though meta-analysis was not performed in that study, the authors

\section{AFFILIATION}

1 Department of Family

Medicine, National Taiwan University Hospital, Taipei

City, Taiwan

2 Department of Family

Medicine, College of

Medicine, National Taiwan

University, Taipei City, Taiwan

CORRESPONDENCE TO

Fei R. Guo. Department of Family Medicine,

National Taiwan University Hospital, No. 7, Zhongshan

South Road, Taipei City 100,

Taiwan. E-mail:

fjguo1@ntu.edu.tw ORCID ID: https://orcid.

org/0000-0003-0671-9529

\section{KEYWORDS}

smoking, COVID-19,

meta-analysis

Received: 25 April 2020 Revised: 30 April 2020 Accepted: 30 April 2020

Figure 1. Forest plot of the updated meta-analysis

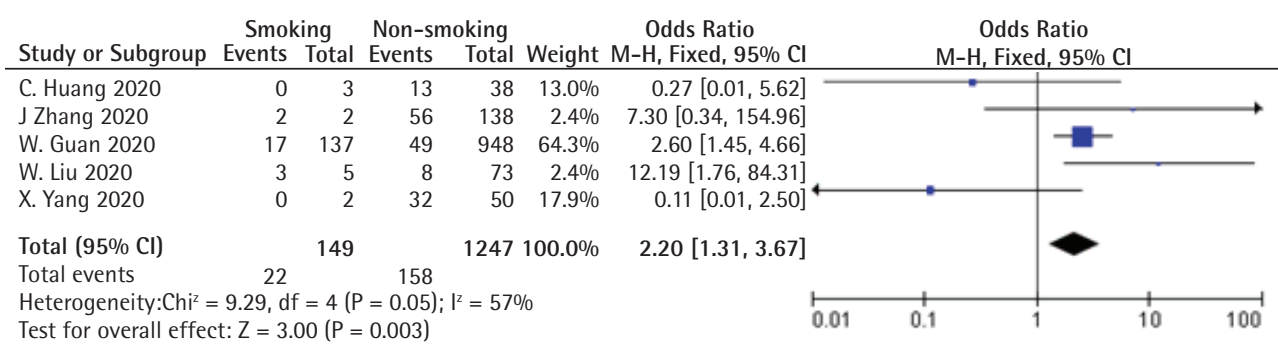


concluded that smoking is most likely associated with the negative progression and adverse outcomes of COVID-19. A recent meta-analysis including 7 studies also revealed that smokers have a double risk of severe COVID-19 (pooled OR=1.98; 95\% CI: 1.29-3.05) ${ }^{8}$. A meta-analysis published in 2019 including 27 studies and 460592 participants revealed current smokers (pooled OR=2.17; 95\% CI: 1.70-2.76) and ex-smokers (pooled OR=1.49; 95\% CI: 1.26-1.75) were more likely to develop community-acquired pneumonia compared to never smokers ${ }^{9}$. The evidence suggests that smokers are more vulnerable to lung infection, and COVID-19 is no exception.

In conclusion, the results of this updated meta-analysis suggest that active smoking is significantly associated with the risk of severe COVID-19. Though more data are available now, they are not included in this study. However, the early meta-analysis of the Lippi and Henry ${ }^{1}$ paper should have had different results.

\section{REFERENCES}

1. Lippi G, Henry BM. Active smoking is not associated with severity of coronavirus disease 2019 (COVID-19). Eur J Intern Med. 2020;75. doi:10.1016/j.ejim.2020.03.014

2. Guan WJ, Ni ZY, Hu Y, et al. Clinical characteristics of coronavirus disease 2019 in China. N Engl J Med. 2020;382(18). doi:10.1056/NEJMoa2002032

3. Huang C, Wang Y, Li X, et al. Clinical features of patients infected with 2019 novel coronavirus in Wuhan, China. Lancet. 2020;395(10223):497-506. doi:10.1016/s0140-6736(20)30183-5

4. Liu W, Tao ZW, Lei W, et al. Analysis of factors associated with disease outcomes in hospitalized patients with 2019 novel coronavirus disease. Chin Med J. 2020;133(9). doi:10.1097/CM9.0000000000000775

5. Yang X, Yu Y, Xu J, et al. Clinical course and outcomes of critically ill patients with SARS-CoV-2 pneumonia in Wuhan, China: a single-centered, retrospective, observational study. Lancet Respir Med. 2020. doi:10.1016/S2213-2600(20)30079-5

6. Zhang JJ, Dong X, Cao YY, et al. Clinical characteristics of 140 patients infected by SARS-CoV-2 in Wuhan, China. Allergy. 2020. doi:10.1111/all.14238

7. Vardavas CI, Nikitara K. COVID-19 and smoking: A systematic review of the evidence. Tob Induc Dis. 2020;18(March). doi:10.18332/tid/119324

8. Zhao Q, Meng M, Kumar R, et al. The impact of COPD and smoking history on the severity of Covid-19: A systemic review and meta-analysis. J Med Virol. 2020. doi:10.1002/jmv.25889

9. Baskaran V, Murray RL, Hunter A, Lim WS, McKeever TM. Effect of tobacco smoking on the risk of developing community acquired pneumonia: A systematic review and meta-analysis. PLoS One. 2019;14(7):e0220204. doi:10.1371/journal.pone.0220204

CONFLICTS OF INTEREST

The author has completed and submitted the ICMJE Form for Disclosure of Potential Conflicts of Interest and none was reported.

\section{FUNDING}

There was no source of funding for this research.

PROVENANCE AND PEER REVIEW

Not commissioned; internally peer reviewed. 